\title{
A Magnetic Coupling Based Strategy for Synchronization of a System Consisting of Chaotic Modified Van der Pol Duffing Oscillators
}

\author{
L. K. Kana, ${ }^{1}$ A. Fomethe, ${ }^{2}$ H. B. Fotsin, ${ }^{1}$ and P. H. Louodop Fotso ${ }^{1}$ \\ ${ }^{1}$ Laboratory of Electronics and Signal Processing, Department of Physics, Faculty of Science, University of Dschang, \\ P.O. Box 67, Dschang, Cameroon \\ ${ }^{2}$ Laboratory of Mechanics and Modeling of Physical Systems, Faculty of Science, University of Dschang, P.O. Box 67, Dschang, Cameroon
}

Correspondence should be addressed to H. B. Fotsin; hbfotsin@yahoo.fr

Received 12 July 2014; Accepted 26 November 2014; Published 28 December 2014

Academic Editor: Thordur Runolfsson

Copyright @ 2014 L. K. Kana et al. This is an open access article distributed under the Creative Commons Attribution License, which permits unrestricted use, distribution, and reproduction in any medium, provided the original work is properly cited.

\begin{abstract}
We propose a new paradigm for the synchronization of two unconnected magnetic core coils based modified Van der Pol Duffing (MVDPD) oscillators circuits. The method is such that only magnetic field based coupling is sufficient to drive two identical chaotic circuits to a synchronized state as well as achieving the global stabilization of the system to its regular dynamics. The dynamics of the coupled system is investigated and Lyapunov stability theory is applied to prove that under some conditions the drive-response system can achieve practical synchronization. Numerical and PSpice simulations are given to demonstrate the effectiveness of the controller.
\end{abstract}

\section{Introduction}

In 1990, the problems of chaos control [1] and synchronization [2] emerged as two exciting topics in nonlinear science with promising applications. Pecora and Carroll [2] introduced a method to synchronize two identical chaotic systems evolving from different initial conditions, while Ott et al. [1] presented the OGY control algorithm. Chaos synchronization is closely related to the observer problem in control theory and recent studies deal with the synchronization problem based on control theory approach. Chaos synchronization has been intensively investigated in the context of many specific problems arising from physical $[3,4]$, chemical, and ecological systems [5] and applications to secure communications [6-8] to just mention a few. Enormous research progress has been made in developing and understanding various types of synchronization schemes, such as adaptive control [9], active-backstepping design [10], active control [11-13], backstepping [14], sliding mode control [15], and self-adaptive backstepping [16].

In most of the methods mentioned above, the controllers and the design approach are often very complex and could be difficult to achieve in practice. Thus, designing simple and available control inputs that can achieve global and stable synchronization of coupled oscillators is generally significant and of practical interest in view of the foreseen applications of chaos synchronization in circuits and lasers. This is an open challenge that has remained unresolved. The synchronization in chaotic circuits coupled by mutual inductors has been extensively studied [17]. To the best of our knowledge, little has been done in the synchronization by magnetic field. In this paper, we propose a new approach of synchronization in chaotic circuits coupled by magnetic field. A magnetic core coil is tuned based on the magnetoimpedance effect [18]. As the bias DC magnetic field is applied along the axis of the inductor body, the transverse permeability of the soft magnetic layer can be changed to achieve the tunability of the inductance. The tunable magnetic core coils have a major role in the current situation where compact designs with high performance are demanded. They are the basic elements of radio frequency that enable the batch fabrication of miniature mechanic structures, devices, and systems for microwave and wireless communication application. The tunable magnetic core coils and capacitors can be used to form tunable filters and low-noise voltage-controlled oscillators (VCOs) [19]. The controller that we propose here depends only on a system 
parameter (the coupling coefficient of the two oscillators coils).

This paper has four aims.

(i) We analyse the dynamics of modified Van der Pol Duffing oscillators coupled by tunable magnetic core coils transformer.

(ii) We use the mutual inductance between the windings (the coupling coefficient) to investigate the synchronization of the two coupled circuits.

(iii) We use the Lyapunov theory, to investigate the conditions leading to practical synchronization of the coupled circuits.

(iv) We present an electronic implementation of this methodology.

The rest of the paper is organized as follows. In Section 2, the circuit of the dynamical systems coupled with a tunable linear transformer is presented, and the appropriate model (sets of nonlinear ordinary differential equations) is derived to describe the dynamical behaviour of the oscillator. In Section 3, basing ourselves on the magnetic coupling factor, we demonstrate the effectiveness of the proposed controller. In Section 4, numerical and experimental simulation results are provided to illustrate the effectiveness and feasibility of the control and synchronization technique. Finally, conclusions are given in Section 5.

\section{Circuit Description and State Equations}

2.1. Circuit Description. Figure 1(a) shows the circuit model of two identical chaotic circuits coupled by a tunable magnetic core coil. Each chaotic subcircuit consists of capacitors, resistors, and a single nonlinear resistor. A physical realization of the cubic nonlinear negative resistor is provided by Figure 1(b); it consists of an operational amplifier (op-amp), four resistors, and ten signal diodes. It is one of the simplest autonomous chaotic circuits.

2.2. State Equations. Let us consider the two magnetic windings of (Figure 1(a)) where $l$ is the lenghth and $r$ is radius. Since $l \ll r$, using Biot-Savart's law, we see that the magnetic field at the center of the winding (1) due to $I_{31}$ is given by

$$
B_{1}=\frac{N_{1} \mu_{0} I_{31}}{2 l}
$$

The flux due to the second winding in the first one is

$$
\phi_{21}=\frac{N_{1} B_{2} S_{1}}{l}\left(1-\frac{2 x^{2}}{l^{2}}\right) .
$$

The mutual inductance is given by

$$
M_{12}=N_{1} \frac{\phi_{21}}{I_{2}} \equiv \sqrt{L \cdot L}\left(1-\frac{2 x^{2}}{l^{2}}\right) .
$$

However, using the reciprocity theorem which combines Amperes's law and Biot-Savart's law, one may show that the mutual inductance is finally

$$
M_{12}=M_{21}=M=\sqrt{L \cdot L}\left(1-\frac{2 x^{2}}{l^{2}}\right)=k \sqrt{L \cdot L},
$$

where $K=\left(1-\left(2 x^{2} / l^{2}\right)\right)$. This result shows that $k$ depends only on the geometrical factors $l$ (length of the coil) and $x$ (distance between the windings) and is independent of the current. The following set of differential equations are obtained by applying Kirchhoff's laws to the circuit shown in Figure 1(a):

$$
\begin{aligned}
c_{1} \frac{d v_{1 j}}{d t} & =\frac{1}{R}\left(v_{2 j}-v_{1 j}\right)-I_{N}\left(v_{1 j}\right), \\
c_{2} \frac{d v_{2 j}}{d t} & =\frac{1}{R}\left(v_{1 j}-v_{2 j}\right)+I_{3 j}, \\
\frac{d i_{3 j}}{d t} & =-\frac{1}{L}\left(v_{2 j}+R_{L} i_{3 j}\right)+\frac{M}{L} \frac{d i_{3(3-j)}}{d t},
\end{aligned}
$$

where $j=1,2 ; M=\sqrt{L \cdot L}\left(1-\left(2 x^{2} / l^{2}\right)\right)=k L ; I\left(v_{1 j}\right)$ represents, respectively, the mutual inductance between the windings and the cubic nonlinear resistor with $v-i$ characteristic $I_{N}\left(v_{1 j}\right)=A v_{1 j}+B v_{1 j}^{3}$ with $A \prec 0$ and $B>0$ [20]. We constructed this circuit with the following values of the parameters: $C_{1}=1 \mathrm{nF} ; C_{2}=10 \mathrm{nF} ; R=3000 \Omega$; $R_{0}=19 \Omega ; L=6 \mathrm{mH}$. For the nonlinear resistors element, we used operational amplifiers (UA741 or equivalent), ten signal diodes (IN4148), and three resistors $\left(R_{1}=4700 \Omega ; R_{2}=\right.$ $2700 \mathrm{~K} \Omega ; R_{3}=1200 \mathrm{~K} \Omega$ ) and we obtain $A \simeq-4.005 \times$ $10^{-4}$ and $B \simeq 29.3 \times 10^{-6}$. With this implementation, the MVDPD circuit can also be seen as a physical realization of the Rayleigh oscillator [21].

In order to compare experimental and numerical results, an appropriate rescaling is introduced. With the corresponding change of variables: $t=R C_{2} \tau ; \dot{x}_{j}=\left(d v_{1 j} / d \tau\right) \sqrt{R B}$; $\dot{y}_{j}=\left(d v_{2 j} / d \tau\right) \sqrt{R B} ; \dot{z}_{j}=\left(d i_{3 i} / d \tau\right) \sqrt{R^{3} B}$, one can rewrite (5) to obtain the following adimensional set of differential equations:

$$
\begin{aligned}
& \dot{x}_{j}=\alpha\left[y_{j}+\mu x_{j}-x_{j}^{3}\right], \\
& \dot{y}_{j}=x_{j}-y_{j}+z_{j}, \\
& \dot{z}_{j}=-\beta y_{j}-\gamma z_{j}+k \dot{z}_{3-j},
\end{aligned}
$$

where $\alpha=C_{2} / C_{1} ; \beta=R^{2} C_{2} / L ; \gamma=R R_{0} C_{2} / L ; \mu=-(1+A R)$; and $0 \leq k \leq 1$.

2.3. Dynamics of the Model. As we mentioned above, one of the main advantages of this circuit for experimental applications is the richness of its dynamics. Here, the types of behaviors are identified using two indicators. The first indicator is the bifurcation diagram, the second being the largest 1D numerical Lyapunov exponent denoted by 


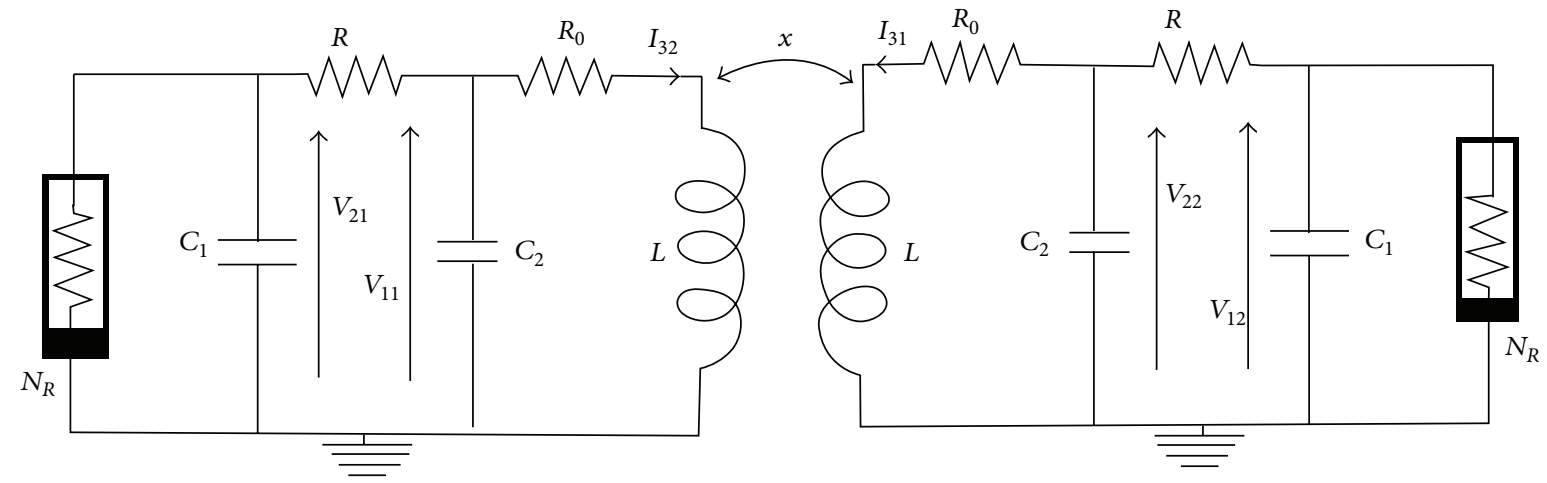

(a)

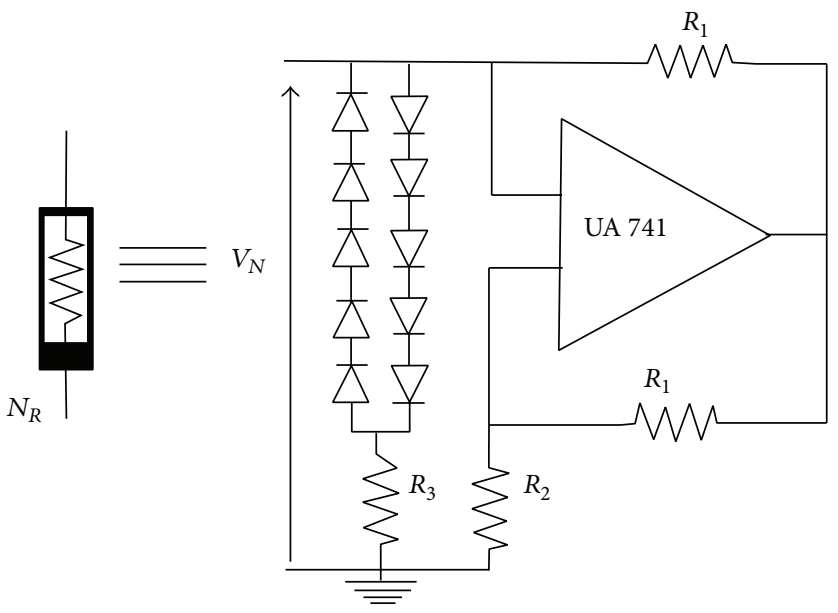

(b)

Figure 1: (a) The electrical model of the system consisting of two mutually coupled modified Van der Pol Duffing oscillators (MVDPD). (b) The practical realization of the electrical circuit corresponding to the cubic nonlinear negative resistance: the operational amplifier can be any compatible TL082; the signal diodes are IN 4148; the circuit elements are $R_{1}=4.7 \mathrm{k} \Omega ; R_{2}=2.7 \mathrm{k} \Omega ; R_{3}=1.2 \mathrm{k} \Omega$.

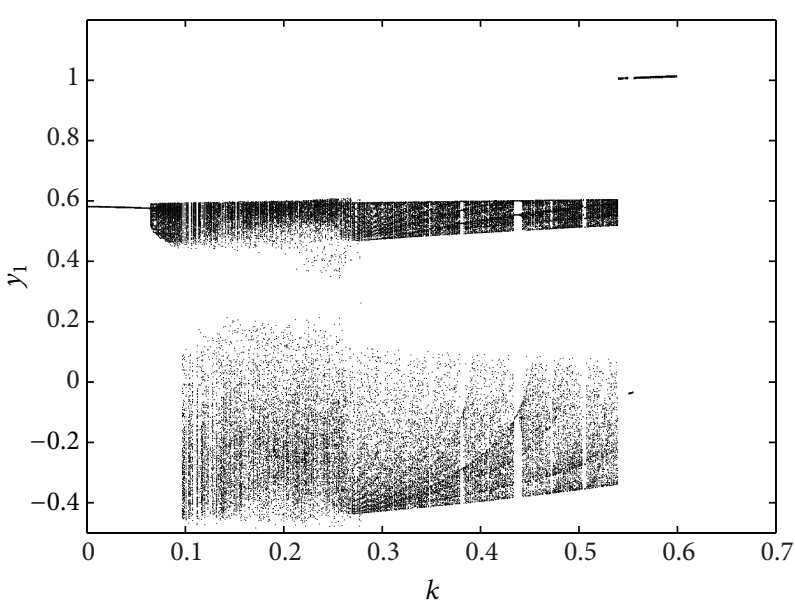

(a)

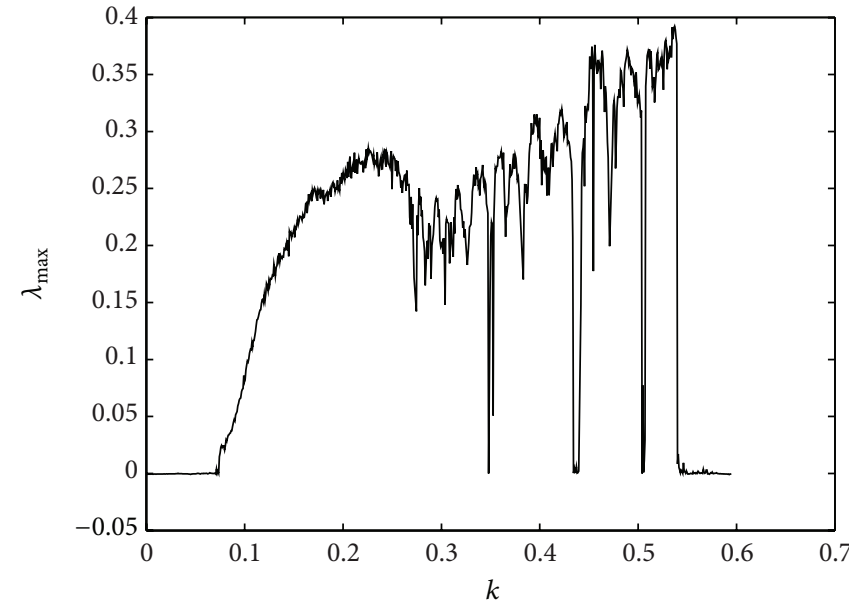

(b)

FIGURE 2: Bifurcation diagram (a) and the graph of 1D largest numerical Lyapunov exponent (b). 
(1)

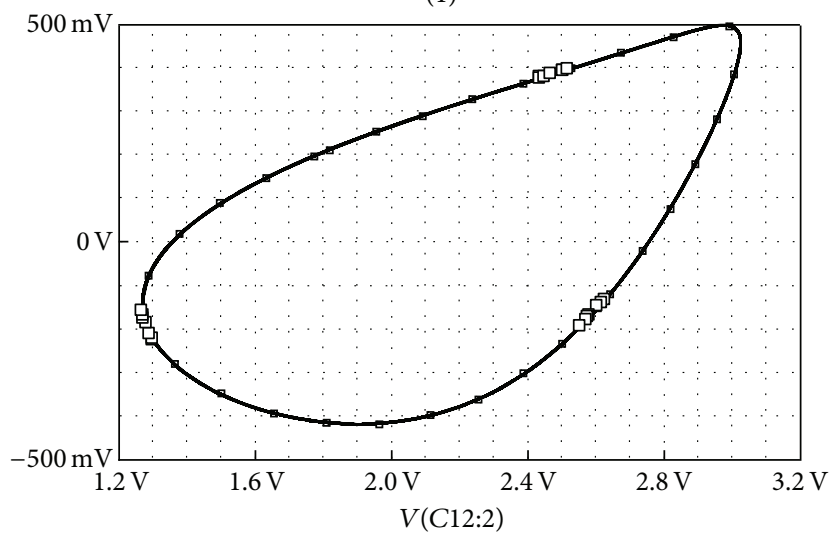

ㅁ $V(C 22: 2)$

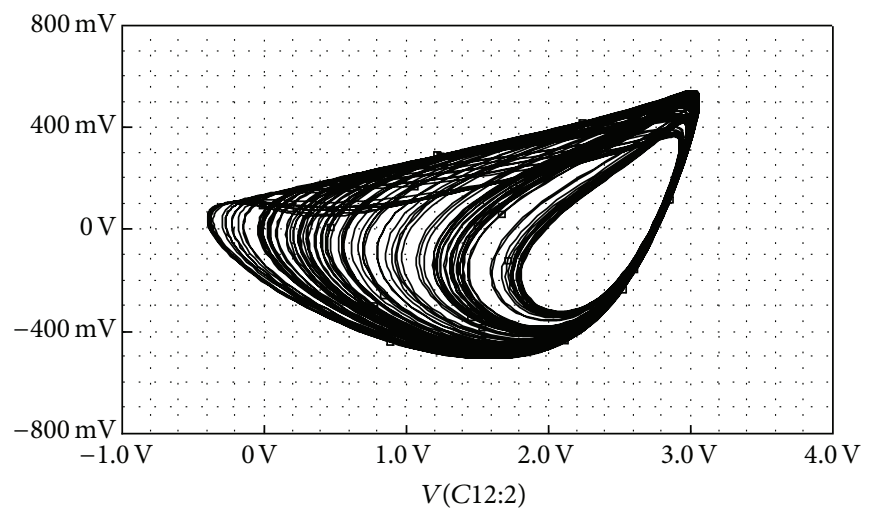

$V(C 22: 2)$

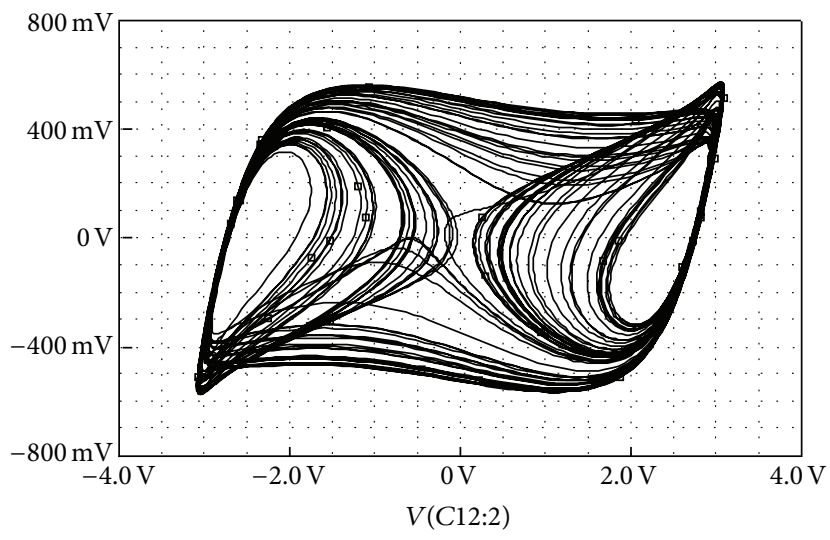

ㅁ $V(C 22: 2)$

(a)

(b)
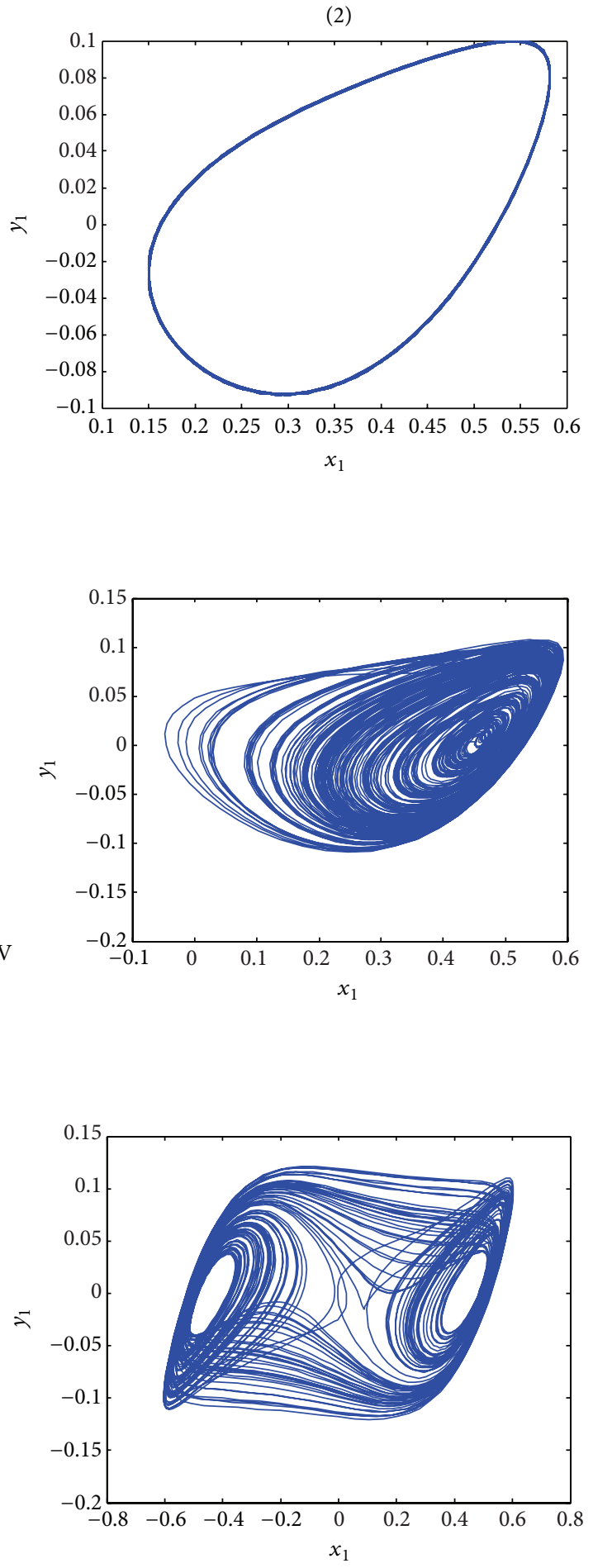

(c)

FIGURE 3: Qualitative comparison of numerical phase portraits forms (2) and experimental (PSpice) phase portraits (1) of the oscillator. 


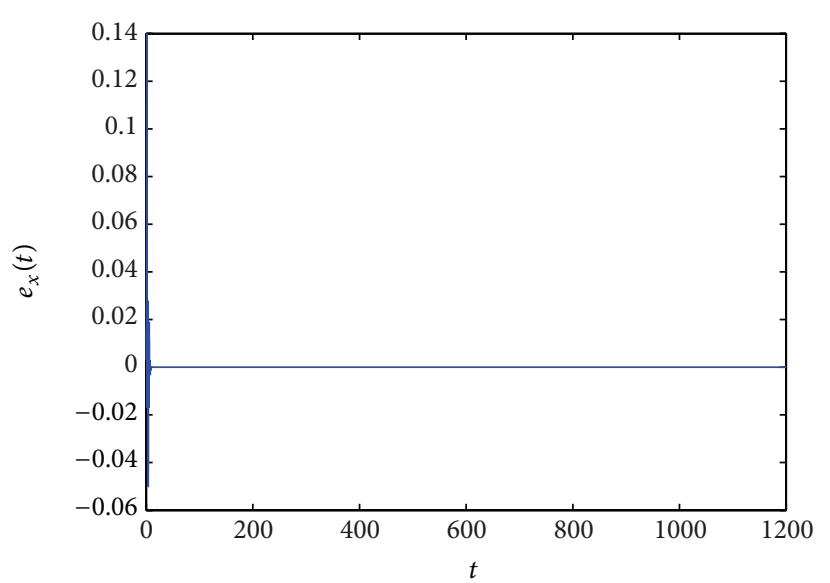

(a)

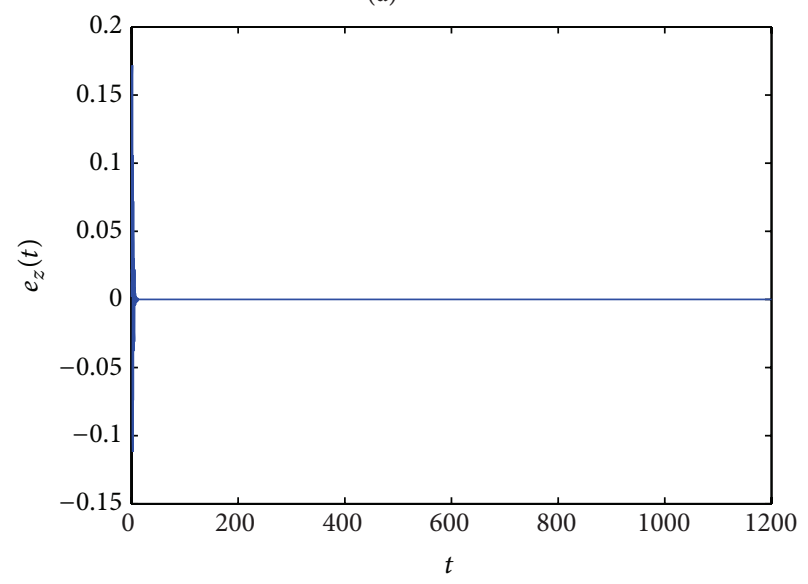

(c)

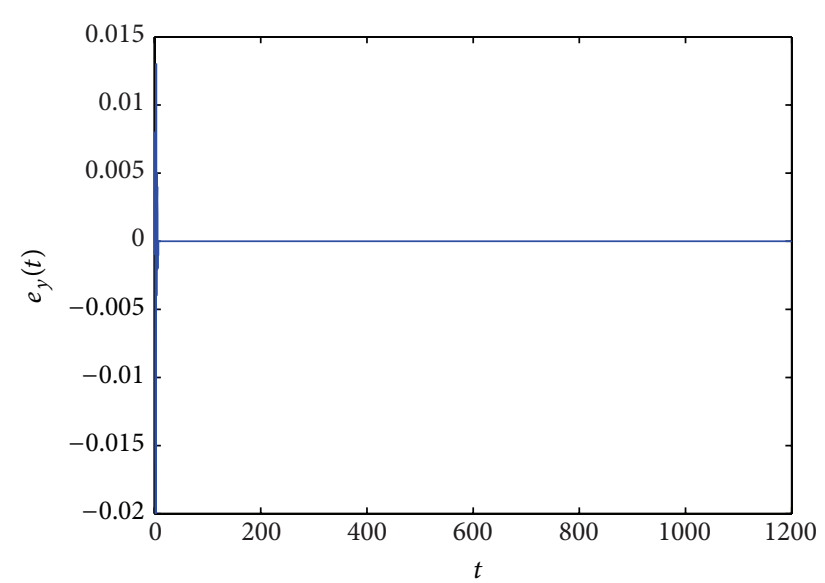

(b)

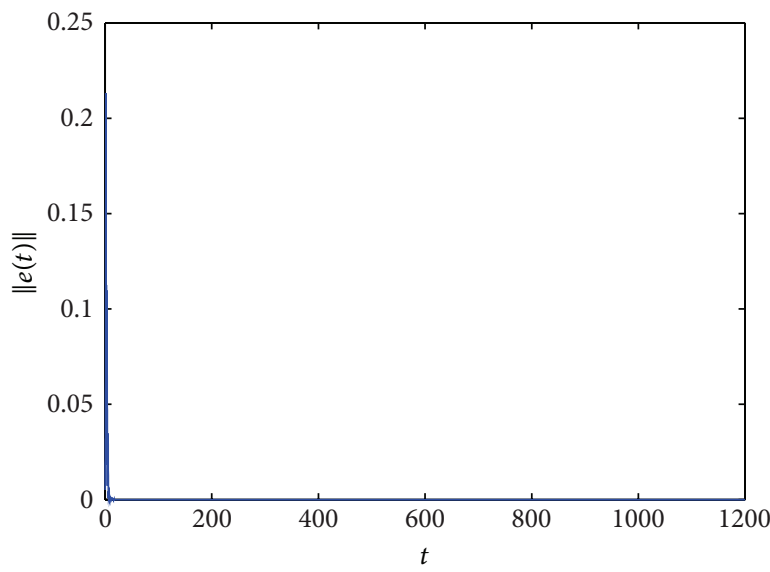

(d)

FIGURE 4: Graphs of time variation of the synchronization errors $e_{x}(t), e_{y}(t)$, and $e_{z}(t)$ and $\|e(t)\|$.

$$
\begin{aligned}
\lambda_{\max } & =\lim _{t \rightarrow \infty}\left[\left(\frac{1}{t}\right) \ln \sqrt{\left(\delta x_{j}\right)^{2}+\left(\delta y_{j}\right)^{2}+\left(\delta z_{j}\right)^{2}}\right] \\
& =\lim _{t \rightarrow \infty}\left[\frac{\ln [d(t)]}{t}\right]
\end{aligned}
$$

and computed from the variational equation obtained by perturbing the solution of (6) as follows: $x \rightarrow x+\delta x$. $d(t)$ is the distance between neighbouring trajectories in the phase space. Asymptotically, $d(t)=e^{\lambda_{\max }}$. Thus, if $\lambda_{\max }>0$, neighbouring trajectories diverge and the state is chaotic. If $\lambda_{\max }<0$, these trajectories converge and the state is nonchaotic. $\lambda_{\max }=0$ for the torus states [21]. Setting $\alpha=10$, $\beta=18, \gamma=0.15$, and $\mu=0.2$, we analyze the effects of the coupling coefficient $k$ (or the distance between the magnetic core coils) on the behavior of the MVDPD oscillators. Therefore, a scanning process is performed to investigate the sensitivity of the oscillators to tiny changes in $k$. The investigation is carried out in the following window: $0<$ $k<0.6$. For the numerical computations of the bifurcation diagrams, the control parameter $k$ is increased from an initial value $k_{i}$ to a final value $k_{f}$ with a step $\Delta k=10^{-4}$. The last computed cyclic point for a given value of $k$ is always used as a new initial value for the next value of $k$. Starting with initial conditions $\left(x_{1}, y_{1}, z_{1}\right)=(0.001,0.004,0.0004)$, $\left(x_{2}, y_{2}, z_{2}\right)=(0.0003,0.00021,0.0004)$ at $k_{i}$, system (6) is integrated, using the standard fourth-order Runge Kutta algorithm. The time step is always $\Delta t=0.005$. A point on the Poincaré cross section is detected after all transient has died out and whenever the trajectory in the phase space $\left(x_{1}, \dot{x}_{1}\right)$ crosses the plane $\left(\dot{x}_{1}=0\right)$ in the negative direction.

Considering the effects of $k$, it appears that the oscillator described by (6) leads to complex dynamical behaviors, such as periodic, multiperiodic, and chaotic states. Figure 2 provides some sample results, showing the bifurcation diagram $\left(k, y_{j}\right)$ (Figure 2(a)) associated with its corresponding graph of largest 1D numerical Lyapunov exponent (Figure 2(b)). Figure 3 shows some numerical phase portraits, respectively, direct numerical (1) and PSpice (2) simulations chosen in the window $0<k \leq 1$. Figure 3(a) shows a periodic attractor $(k=0.1)$; Figures $3(\mathrm{~b})$ and $3(\mathrm{c})$ show chaos $(k=0.3$ and $k=0.44)$. Practically, the distance between two magnetically coupled MVDP systems or the position or type of magnetic coupling core could have drastic effects on the oscillators dynamics, even if the number of oscillators is increased. This is an interesting behaviour in the sense of control of the collective dynamics of magnetically coupled nonlinear oscillators. 


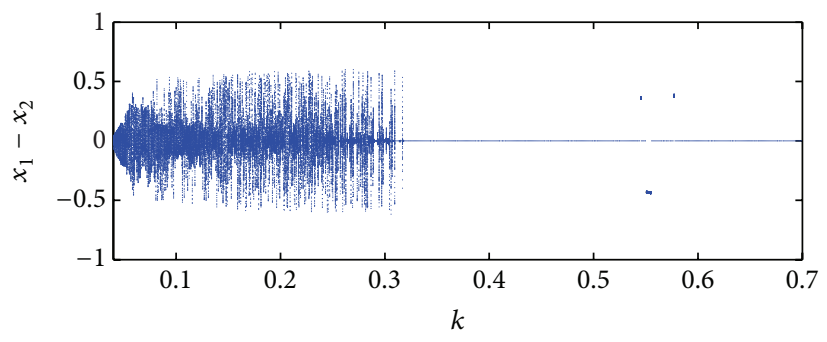

(a)

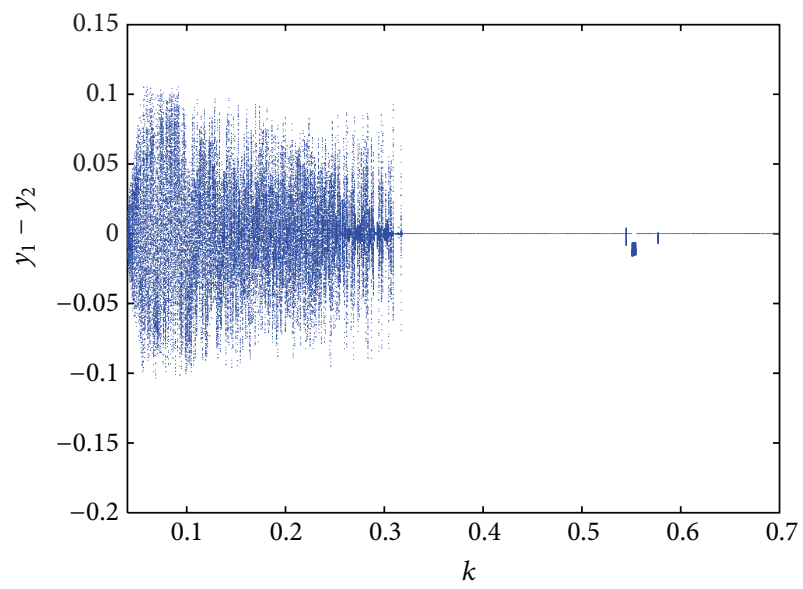

(b)

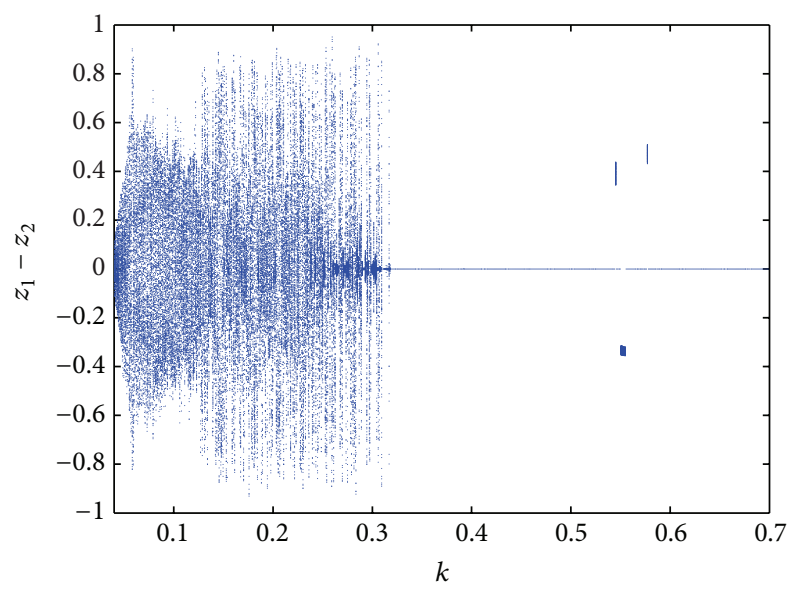

(c)

FIGURE 5: Bifurcation diagram for the in-phase synchronization.

\section{Synchronization Scheme}

Let us consider here that the drive system is given by the following set of coupled differential equations:

$$
\begin{aligned}
& \dot{x}_{1}=\alpha\left[y_{1}+\mu x_{1}-x_{1}^{3}\right], \\
& \dot{y}_{1}=x_{1}-y_{1}+z_{1}, \\
& \dot{z}_{1}=-\beta y_{1}-\gamma z_{1}+k \dot{z}_{2} .
\end{aligned}
$$

According to our aim, we define the controlled response system as the following set of differential equations:

$$
\begin{aligned}
& \dot{x}_{2}=\alpha\left[y_{2}+\mu x_{2}-x_{2}^{3}\right], \\
& \dot{y}_{2}=x_{2}-y_{2}+z_{2}, \\
& \dot{z}_{2}=-\beta y_{2}-\gamma z_{2}+k \dot{z}_{2},
\end{aligned}
$$

where $k$ is the control parameter, which could be the distance between the two coils or the magnetic parameters of the core (tunable transformer) [19]. We define the error system as the difference between the signals from drive and the response system as $e_{x}=x_{1}-x_{2}, e_{y}=y_{1}-y_{2}$, and $e_{z}=z_{1}-z_{2}$. By considering the time derivatives of the error signals together with (8) and (9), we obtain the error dynamics system:

$$
\dot{e}_{x}=\alpha\left[e_{y}+\mu e_{x}-e_{x}^{3}-3 x_{1} x_{2} e_{x}\right],
$$

$$
\begin{aligned}
& \dot{e}_{y}=e_{x}-e_{y}+e_{z}, \\
& \dot{e}_{z}=-\beta_{0} e_{y}-\gamma_{0} e_{z},
\end{aligned}
$$

where $\beta_{0}=\beta /(1+k)$ and $\gamma_{0}=\gamma /(1+k)$.

Proof. At this stage, we need to prove that the dynamics of the error system equation (10) practically converges to a limit value, in the sense that when $t \rightarrow \infty,\|e(t)\| \rightarrow \xi$, where $\xi$ is a positive small constant. This can be qualitatively analyzed with Lyapunov stability theory as follows. Consider a Lyapunov function candidate in the following form:

$$
\begin{aligned}
V= & \frac{1}{2}\left[\frac{e_{x}^{2}}{\alpha}+e_{y}^{2}+\frac{e_{z}^{2}}{\beta_{0}}\right] \\
& +\int_{0}^{t}\left[\left(e_{x}^{2}-1\right)^{2}+\left(1-\mu+3 x_{1} x_{2}\right) e_{x}^{2}\right] d \tau .
\end{aligned}
$$


(1)
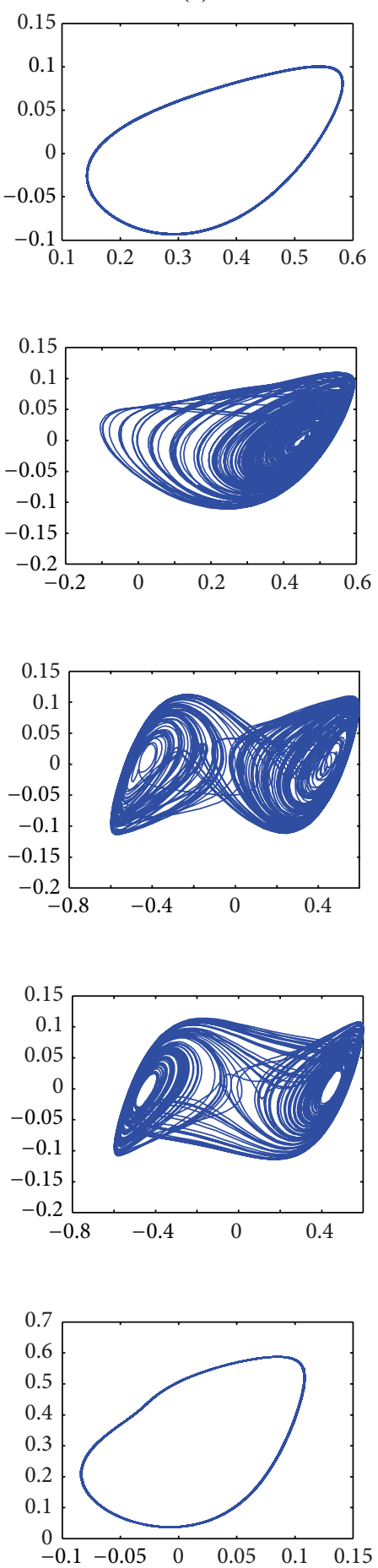

(2)

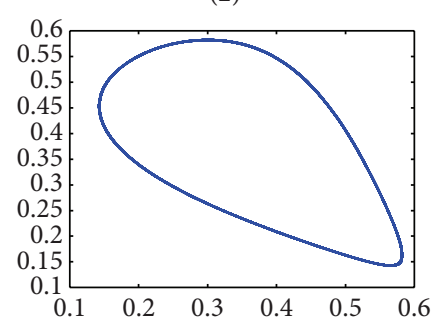

(a)

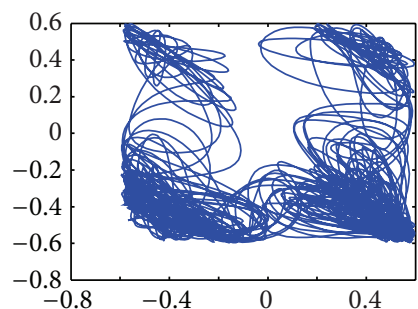

(b)

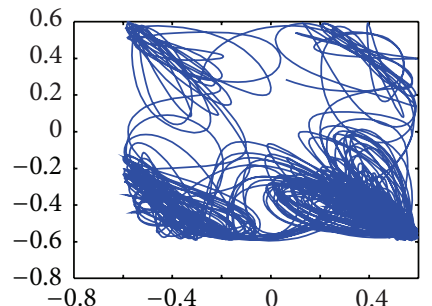

(c)

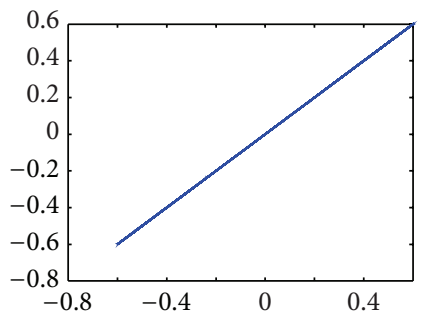

(d)

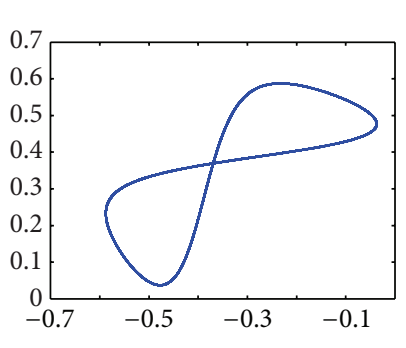

(e)
(3)
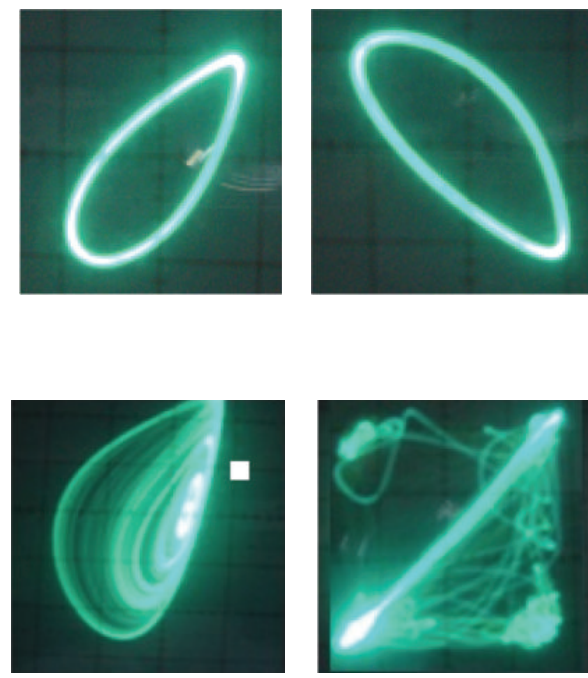

(4)

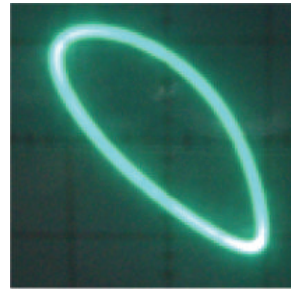

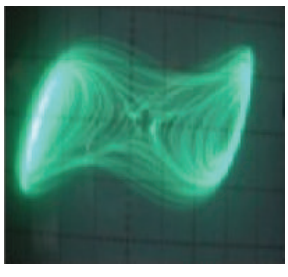
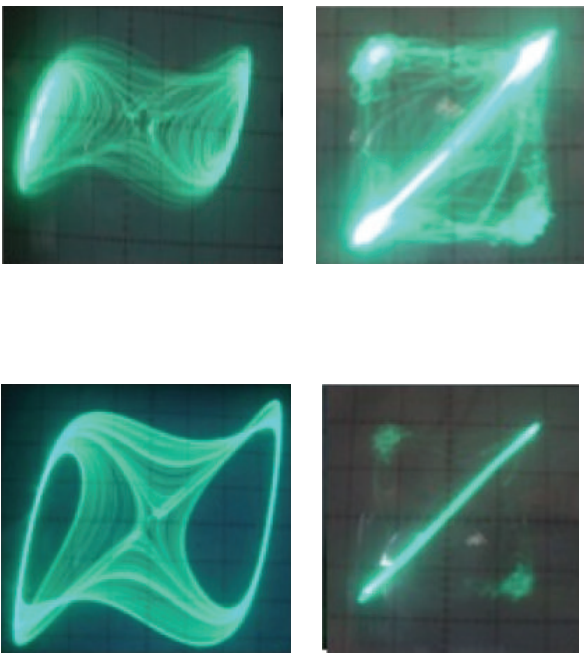
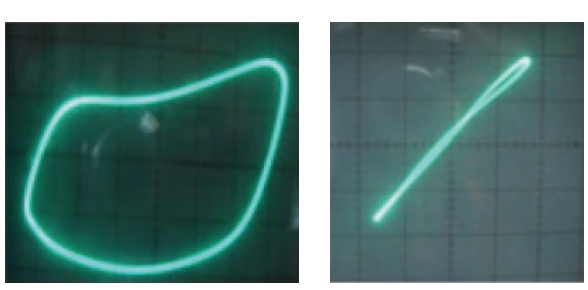

FIGURE 6: In-phase synchronization of the magnetically coupled chaotic circuits (a) $k=0$; (b) $k=0.1$; (c) $k=0.2$; (d) $k=0.4$; and (e) $k=0.54$. (1) $x_{1}$ versus $y_{1}$; (2) $x_{1}$ versus $x_{2}$; (3) $V_{11}$ versus $V_{12}$; (4) $V_{11}$ versus $V_{21}$.

The time derivative of this function with respect to the system of errors equations (10) satisfies

$$
\dot{V}=-\left(e_{x}-e_{y}\right)^{2}-\frac{\gamma_{0}}{\beta_{0}} e_{z}^{2}-(\varphi(0)-1-\mu) e_{x}^{2}(0)
$$

with $\varphi(0)=e_{x}^{2}(0)-3 x_{1}(0) x_{2}(0)$.
In this case, (12) leads to

$$
\dot{V} \leq(-\varphi(0)+1+\mu) e_{x}^{2}(0)
$$

if $\varphi(0) \leq 1+\mu$ the Lyapunov function is contained in a small ball with radius $r \leq 1+\mu$. 
If $\varphi(0) \geq 1+\mu$, then $\dot{V} \leq \phi$ which is a positive constant. Now, we will estimate the settling time $\tau_{s}$ using the approach developed in [22]. Integrating (13) from 0 to $\tau_{s}$ gives

$$
V\left(\tau_{s}\right)-V(0) \leq(-\varphi(0)+1+\mu) e_{x}^{2}(0) \tau_{s}
$$

Using the fact that $V\left(\tau_{s}\right) \rightarrow 0$ when $\tau \rightarrow \tau_{s}$ and $V(\tau)>0$ for all $\tau$ and taking into account (13), it appears that the Lyapunov function $V(\tau)$ is a monotonous and decreasing function. Hence, it follows that

$$
-V(0) \leq V\left(\tau_{s}\right)-V(0) .
$$

Thus, one obtains the following that presents the bounds of the Lyapunov function $V(\tau)$ :

$$
-V(0) \leq V\left(\tau_{s}\right)-V(0) \leq(-\varphi(0)+1+\mu) e_{x}^{2}(0) \tau_{s} .
$$

Hence, one has

$$
\begin{aligned}
\tau_{s} & =\frac{V(0)}{(-\varphi(0)+1+\mu) e_{x}^{2}(0)} \\
& =\frac{1}{\alpha \beta e_{x}^{2}}\left(\frac{\beta e_{x}^{2}+\alpha \beta e_{y}^{2}+\alpha e_{z}^{2}}{-e_{x}^{2}(0)+3 x_{1}(0) x_{2}(0)+1-\mu}\right) .
\end{aligned}
$$

Relation (17) gives the maximum settling time for synchronization. Hence, the finite-time synchronization of two identical oscillators is reached when the numerical settling time $\tau_{\mathrm{Nu}}$ satisfies the relation $\tau_{\mathrm{Nu}} \prec \tau_{\mathrm{Th}}$, where

$$
\tau_{\mathrm{Th}}=\frac{1}{\alpha \beta e_{x}^{2}}\left(\frac{\beta e_{x}^{2}+\alpha \beta e_{y}^{2}+\alpha e_{z}^{2}}{-e_{x}^{2}(0)+3 x_{1}(0) x_{2}(0)+1-\mu}\right) .
$$

This concludes the proof.

\section{Numerical and Experimental Simulations}

We now present numerical simulation results to verify the effectiveness of the controller. In all cases, we select the parameters $\alpha=10, \beta=18, \gamma=0.15, \mu=0.2$, and $k=$ 0.44 such that the chaotic state in Figure 3 is maintained and choose the following initial conditions for the drive-response system: $\left(x_{1}, y_{1}, z_{1}\right)=(0.001,0.004,0.0004)$ and $\left(x_{2}, y_{2}, z_{2}\right)=$ $(0.003,0.0021,0.0004)$.

In Figure 4, we illustrate the performance of the controller. The error dynamics is found to converge to the zero solution as $t \rightarrow \infty$, implying that the synchronization between systems (8) and (9) has been achieved. In Figure 4, we present the time evolutions of the synchronization errors and their norms. According to these graphs, one observes that the synchronization is reached, as the errors amplitudes are decreasing with time towards a small limit close to zero. The graphs of Figure 5 show the synchronization boundaries of the system according to the coupling parameter $k$.

The experimental simulation is a nice way to scan the parameter range in order to find the proper parameter values for a numerical simulation. Another advantage of such an implementation with respect to numerical simulation is that there is no need to wait for long transient times. This justifies the increasing interest devoted to this type of implementation for the analysis of nonlinear and chaotic physical systems. Our aim in this section is to consider and implement an appropriate analog real circuit for the investigation of the system described by (6). We here restrict our analysis to the study of different bifurcations and synchronization states of the system. In the following circuit experiments, the values of the parameters are $C_{1}=1 \mathrm{nF} ; C_{2}=10 \mathrm{nF} ; R=3 \mathrm{k} \Omega$; $R_{0}=19 \Omega ; L=6 \mathrm{mH}$.

Figure 6 shows in-phase synchronization of period-one attractors. As the parameter $k$ increases, one periodic attractor bifurcates to chaotic attractor via torus route keeping inphase synchronization.

\section{Conclusion}

In this paper, we have proposed a new paradigm for the synchronization of two unconnected magnetic core coils based modified Van der Pol Duffing (MVDPD) oscillators circuits. The method is such that only magnetic field based coupling is sufficient to drive two identical chaotic circuits to a synchronized state as well as achieving the global stabilization of the system to its regular dynamics. The dynamics of the coupled system was investigated and Lyapunov stability theory was applied to prove that under some conditions the drive-response system can achieve practical synchronization. Numerical and experiment simulations were given to demonstrate the effectiveness of the controller. In future works, it will be shown that collective dynamics of several magnetically coupled MVDPD systems could be controlled by moving a transformer's core or varying distances between oscillators.

\section{Conflict of Interests}

The authors declare that there is no conflict of interests regarding the publication of this paper.

\section{References}

[1] E. Ott, C. Grebogi, and J. A. Yorke, “Controlling chaos," Physical Review Letters, vol. 64, no. 11, pp. 1196-1199, 1990.

[2] L. M. Pecora and T. L. Carroll, "Synchronization in chaotic systems," Physical Review Letters, vol. 64, no. 8, pp. 821-825, 1990.

[3] M. Lakshmanan and K. Murali, Chaos in Nonlinear Oscillators: Controlling and Synchronizing, World Scientific, 1996.

[4] U. E. Vincent, A. N. Njah, O. Akinlade, and A. R. Solarin, "Phrase synchronization in unidirectionally coupled chaotic ratchets," Chaos, vol. 14, no. 4, pp. 1018-1025, 2004.

[5] S. K. Han, C. Kurrer, and Y. Kuramoto, "Dephasing and bursting in coupled neural oscillators," Physical Review Letters, vol. 75, no. 17, pp. 3190-3193, 1995.

[6] K. M. Cuomo and A. V. Oppenheim, "Circuit implementation of synchronized chaos with applications to communications," Physical Review Letters, vol. 71, no. 1, pp. 65-68, 1993.

[7] L. Kocarev and U. Parlitz, "General approach for chaotic synchronization with applications to communication," Physical Review Letters, vol. 74, no. 25, pp. 5028-5031, 1995. 
[8] G. J. Fodjouong, H. B. Fotsin, and P. Woafo, "Synchronizing modified van der Pol-Duffing oscillators with offset terms using observer design: application to secure communications," Physica Scripta, vol. 75, no. 5, pp. 638-644, 2007.

[9] H. B. Fotsin and P. Woafo, "Adaptive synchronization of a modified and uncertain chaotic van der Pol-Duffing oscillator based on parameter identification," Chaos, Solitons \& Fractals, vol. 24, no. 5, pp. 1363-1371, 2005.

[10] H. Zhang, X.-K. Ma, Y. Yang, and C.-D. Xu, "Generalized synchronization of hyperchaos and chaos using active backstepping design," Chinese Physics, vol. 14, no. 1, pp. 86-94, 2005.

[11] E.-W. Bai and K. E. Lonngren, "Synchronization of two Lorenz systems using active control," Chaos, Solitons and Fractals, vol. 8, no. 1, pp. 51-58, 1997.

[12] M.-C. Ho and Y.-C. Hung, "Synchronization of two different systems by using generalized active control," Physics Letters A, vol. 301, no. 5-6, pp. 424-428, 2002.

[13] U. E. Vincent, "Synchronization of Rikitake chaotic attractor using active control," Physics Letters A, vol. 343, no. 1-3, pp. 133138, 2005.

[14] X. Tan, J. Zhang, and Y. Yang, "Synchronizing chaotic systems using backstepping design," Chaos, Solitons \& Fractals, vol. 16, no. 1, pp. 37-45, 2003.

[15] M. Jang, C. Chen, and C. Chen, "Sliding mode control of chaos in the cubic Chua's circuit system," International Journal of Bifurcation and Chaos, vol. 12, no. 6, pp. 1437-1449, 2002.

[16] U. E. Vincent, R. K. Odunaike, J. A. Laoye, and A. A. Gbindinninuola, "Adaptive backstepping control and synchronization of a modified and chaotic van der Pol-Duffing oscillator," Journal of Control Theory and Applications, vol. 9, no. 2, pp. 273-277, 2011.

[17] Y. Komatsu, Y. Uwate, and Y. Nishio, "Synchronization in Chaotic circuits coupled by mutual inductors," in Proceedings of the RISP International Workshop on Nonlinear Circuits and Signal Processing (NCSP '06), vol. 6, pp. 293-296, Honolulu, Hawaii, USA, March 2006.

[18] N. Ning, X. P. Li, J. Fan et al., "A tunable magnetic inductor," IEEE Transactions on Magnetics, vol. 42, no. 5, pp. 1585-1590, 2006.

[19] B. Georgescu, H. Pekau, J. Haslett, and J. McRory, "Tunable coupled inductor Q-enhancement for parallel resonant LC tanks," IEEE Transactions on Circuits and Systems II: Analog and Digital Signal Processing, vol. 50, no. 10, pp. 705-713, 2003.

[20] G. P. King and S. T. Gaito, "Bistable chaos. I. Unfolding the cusp," Physical Review A, vol. 46, no. 6, pp. 3092-3099, 1992.

[21] J. C. Chedjou, L. K. Kana, I. Moussa, K. Kyamakya, and A. Laurent, "Dynamics of a quasiperiodically forced rayteigh oscillator," Journal of Dynamic Systems, Measurement and Control, Transactions of the ASME, vol. 128, no. 3, pp. 600-607, 2006.

[22] V. Sundarapandian, "Global chaos synchronization of Arneodo and Shimizu-Morioka chaotic systems by active nonlinear control," International Journal of Advances in Science and Technology, vol. 2, no. 6, pp. 32-42, 2011. 

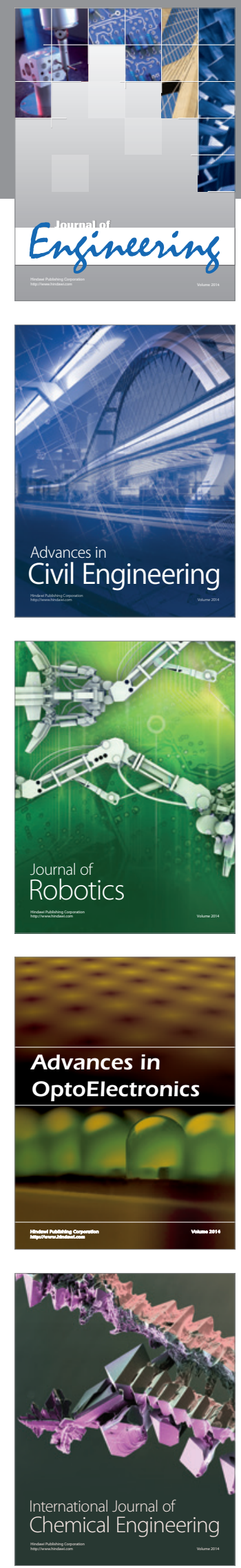

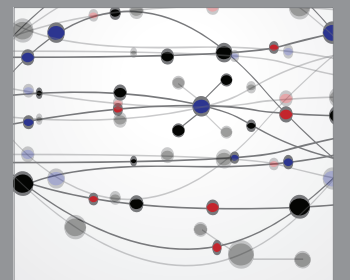

The Scientific World Journal
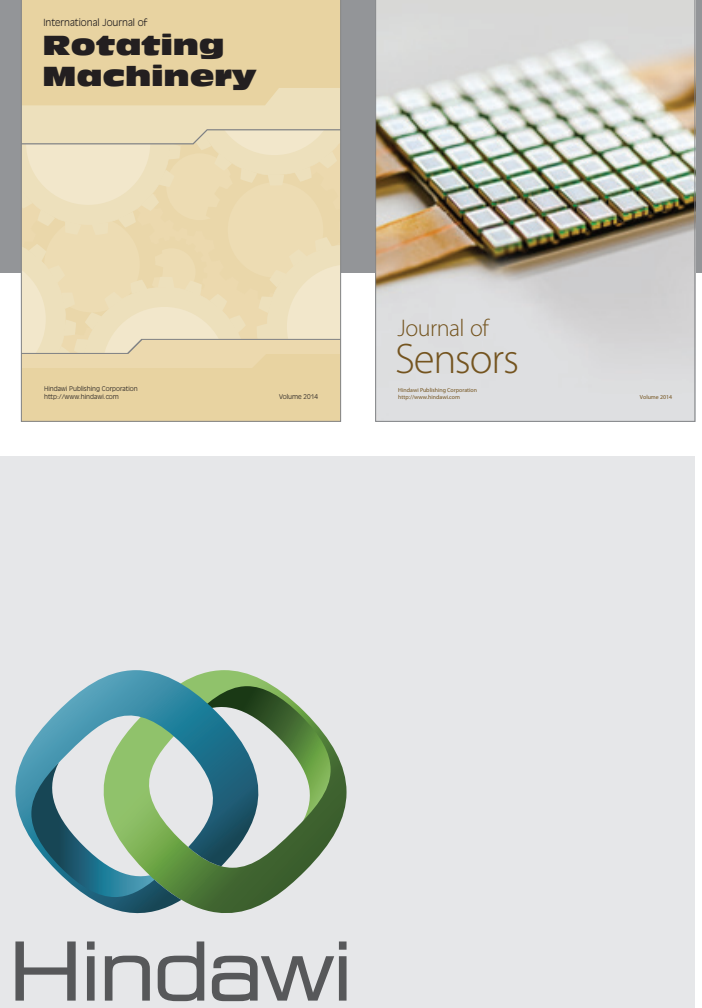

Submit your manuscripts at http://www.hindawi.com
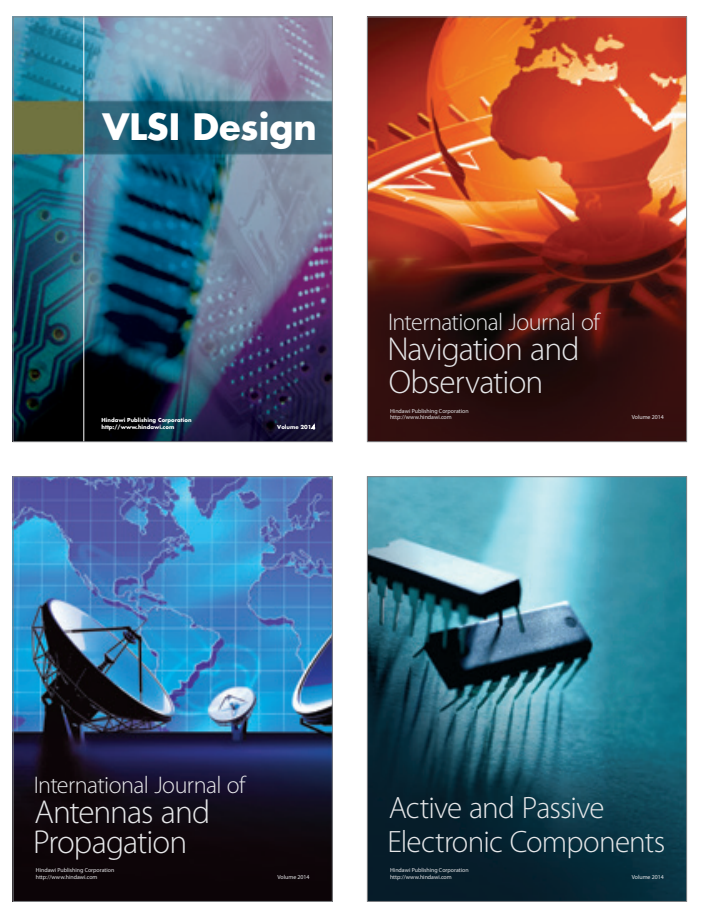
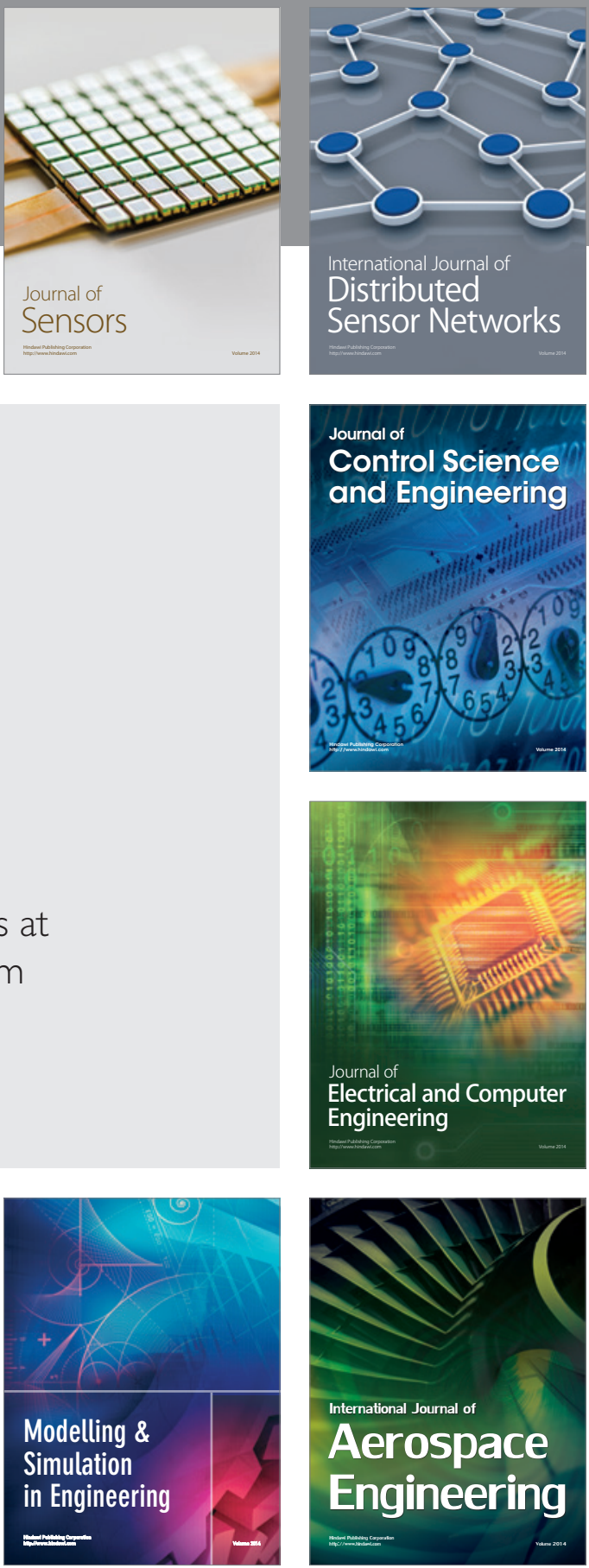

Journal of

Control Science

and Engineering
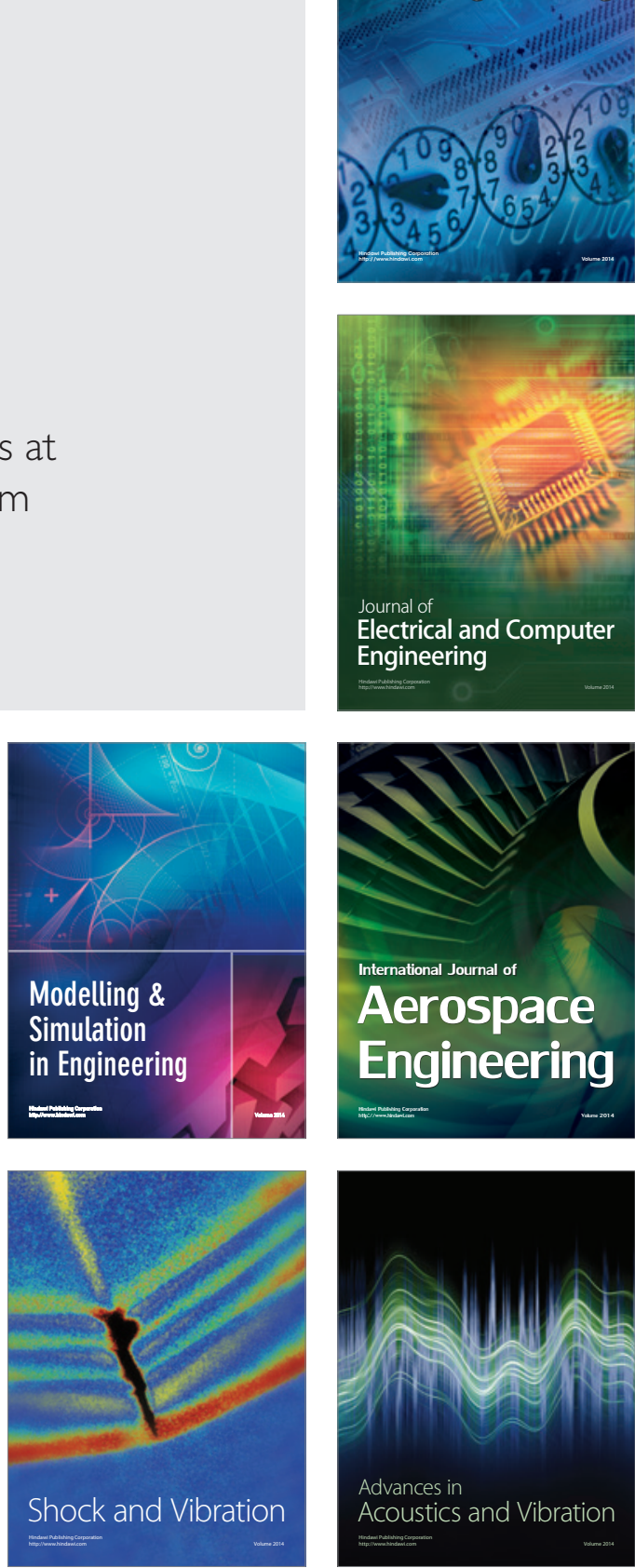